\title{
Recursos educativos en Ciencias de la Salud
}

\section{Educational Resources in Health Sciences}

Jorge Palés ${ }^{\mathrm{a}, \mathrm{b}}$ y Arcadi Guala,b,c

${ }^{a}$ Departamento de Ciencias Fisiológicas I. Facultad de Medicina. Universidad de Barcelona, Barcelona.

${ }^{\mathrm{b}}$ Sociedad Española de Educación Médica. ${ }^{\mathrm{I}}$ Instituto de Salud Carlos III, Madrid.

Este artículo resume la presentación realizada el día 26 de enero de 2004, en el marco del curso de Formación en Metodología de la Investigación organizado por el Instituto de Salud Carlos III. En concreto se revisan los aspectos a tener en cuenta al elaborar un programa formativo como son el proceso de definición de competencias, la elaboración de un programa docente, la elección de las metodologías y estrategias docentes y el rol del profesor.

Palabras clave: Competencias, educación basada en competencias, educación médica basada en la mejor evidencia, roles del profesor
This report summarizes the presentation developed at the Instituto de Salud Carlos III, in Madrid, in January 2004, in a Research Methodology course. Elements as the definition of learning outcomes, the educational program development, the election of the best teaching and learning methods and the new roles of teachers are revised.

Key Words: Outcomes, Outcome-based education, best evidence-based medical education, teacher's roles

\section{INTRODUCCIÓN}

Este artículo resume los principales aspectos de la presentación realizada el día 26 de enero de 2004 , en el marco del curso de Formación en Metodología de la Investigación organizado por el Instituto de Salud Carlos III.

Al plantearnos desarrollar un curso con fines formativos, sea cual sea su temática y sean cuales sean los destinatarios del mismo, es obligado considerar previamente diferentes aspectos desde el punto de vista educativo. Estos aspectos son: a) en primer

Correspondencia:

Jorge Palés

Departamento de Ciencias Fisiológicas

Facultad de Medicina. Universidad de Barcelona

Casanova 143, 08036 Barcelona

Tef. 34934024519

Fax: 34934035295

E-mail: jpales@ub.edu lugar hemos de abordar la definición de competencias que se pretende que los alumnos hayan adquirido al final del programa de formación, b) en segundo lugar nos hemos de plantear la elaboración del programa docente y sus contenidos que deberán estar de acuerdo con las competencias definidas previamente y c) debemos elegir las estrategias y metodologías docentes más adecuadas, incluyendo las metodologías de evaluación. En este artículo nos referiremos esencialmente a estos apartados y finalmente haremos algunas consideraciones sobre el papel que ha de jugar el profesor en el proceso.

\section{DEFINICIÓN DE COMPETENCIAS}

Diversos documentos de índole internacional han expresado desde hace tiempo la necesidad de que al elaborar un programa educativo se expliciten previamente y claramente las competencias que pretendemos que nuestros alumnos adquieran a lo largo 


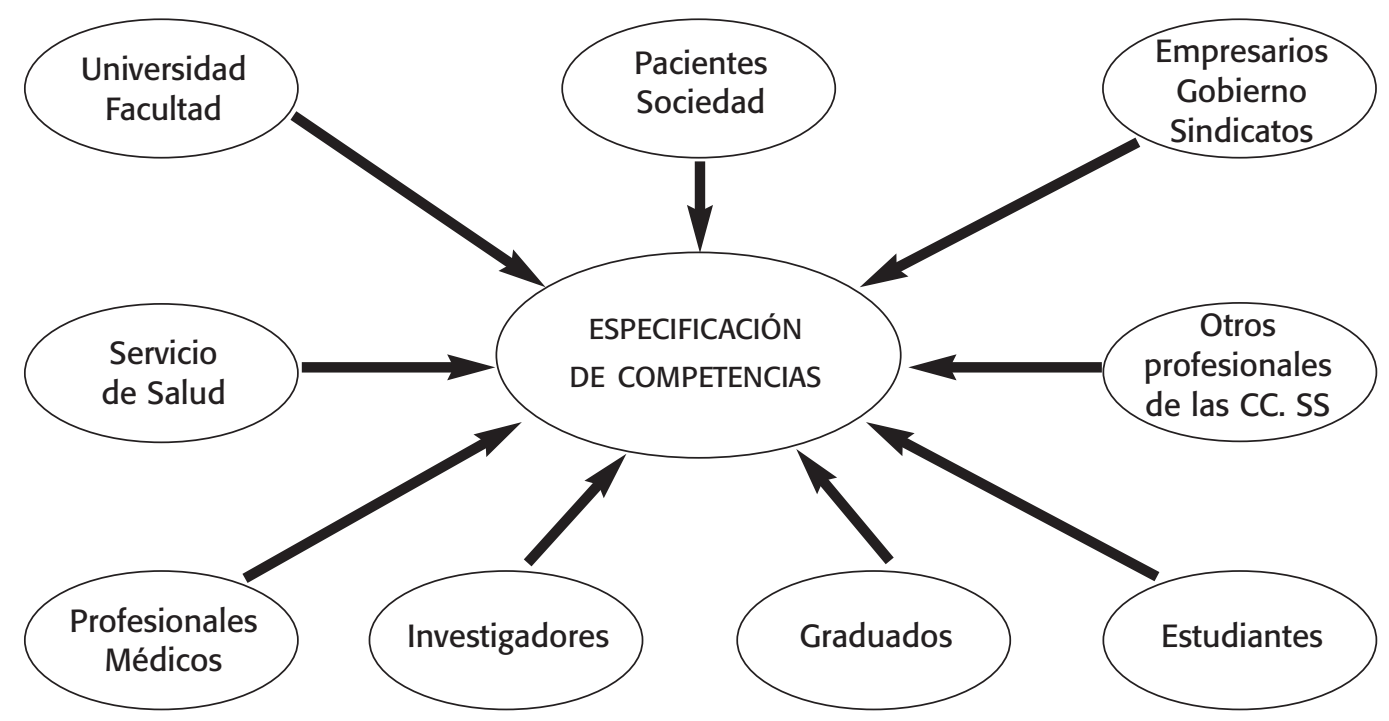

del proceso de aprendizaje. Uno de los documentos que más claramente expresa esta condición son los Estándares de Calidad para la Educación Médica ${ }^{1}$ en sus diferentes etapas, pregrado, postgrado y formación continua, definidos por la World Federation for Medical Education, (WFME). Concretamente, en el capítulo de los Estándares de Calidad para la Educación Médica de Postgrado, la WFME expresa los siguientes requerimientos:

- Las autoridades competentes DEBEN definir, consultadas las organizaciones profesionales, la misión y los resultados que se esperan para los diferentes tipos de formación médica de postgrado, y hacerlos públicos.

- La formulación de la misión y de los resultados DEBE describir el proceso educativo del que ha de resultar un investigador competente para ejercer una actividad investigadora amplia y actualizada en el campo de las ciencias de la salud, sin supervisión ya sea de forma independiente ya sea en el seno de un equipo, y de acuerdo con las funciones que los investigadores puedan ejercer en el sistema sanitario.

- Las competencias se pueden definir en términos profesionales amplios como un conocimiento específico, habilidades, actitudes y comportamientos.

- La formulación de la misión y de los resultados de la formación de postgrado DEBE ser hecha por sus agentes principales.

La definición de competencias debe ir precedida de un estudio previo de las necesidades de la comunidad o población en la que debe realizarse el curso en cuestión. En este caso sería conveniente analizar cuidadosamente cuales son las necesidades en investigación de la comunidad o institución, así como las líneas prioritarias de investigación de las mismas, donde deberían luego desarrollar su actividad los alumnos, al acabar su período de formación.

La especificación de las competencias no es una exclusiva de un determinado grupo (por ejemplo las universidades) sino que en él, deben participar todos los agentes implicados de una forma u otra en los resultados del proceso. Los posibles agentes implicados se exponen en la figura 1. Es obvio que en función de las peculiaridades de cada región o país, podrían añadirse otros posibles agentes.

Existen diferentes modelos para definir las competencias. Aunque estos modelos se refieren básicamente a la formación de pregrado, se pueden aplicar con ligeras modificaciones a otras etapas de la formación médica y como no a un programa de formación en metodología de la investigación. Siguiendo el modelo de Harden ${ }^{2,3}$, los tres ámbitos en los que se han definir competencias serían:

a) Competencias técnicas: aquello que el investigador ha ser capaz de hacer

b) Competencias intelectuales, emocionales, analíticas y creativas: como aborda el investigador su práctica.

c) Competencias personales: el investigador como profesional

Las competencias técnicas incluirían aspectos como habilidades y procedimientos prácticos, habilidades para buscar información, habilidades de comunicación (capacidad para escribir artículos científicos, para expresarse en público), etc.

Las competencias intelectuales, analíticas y crea- 
tivas incluirían entre otros aspectos, las bases teóricas y conocimientos necesarios para llevar a cabo sus tareas, las actitudes adecuadas y la capacidad para tomar decisiones y razonar, etc.

Finalmente el tercer grupo de competencias, las personales, abarcarían los aspectos que se refieren a las funciones del investigador en el sistema universitario o sanitario donde se encuentra y que incluirían el conocimiento del propio sistema y sus peculiaridades, su propio papel como investigador, como docente y gestor de los recursos disponibles y como miembro de equipos multiprofesionales. Por otra parte dentro de este apartado se podrían considerar las competencias de desarrollo personal como podrían ser la capacidad de autocrítica y autoevaluación, la capacidad de aprender autónomamente, la motivación y la capacidad para identificar sus objetivos profesionales a corto y largo plazo y sus aspiraciones personales.

\section{ELABORACIÓN DEL PROGRAMA DOCENTE Y SUS CONTENIDOS}

La definición de las competencias debe ir seguida de la elaboración del programa docente y de la asignación de las diferentes tareas a los diferentes módulos o unidades de que constará el programa, explicitando de que competencias va a responsabilizarse cada una de las unidades o módulos.

La elaboración de un programa educativo debe hacerse por tanto en base a las competencias finales a obtener o en los resultados finales (outcome-based education en terminología inglesa). La educación basada en los resultados es un instrumento de planificación curricular que nos pone de manifiesto que el producto que queremos define el proceso de producción y no a la inversa. ${ }^{4,5}$

De forma resumida podríamos decir que la educación basada en el producto o en los resultados: a) pone énfasis en el producto final, b) ayuda a distinguir lo que es esencial de lo que no es esencial, c) determina lo que se ha de enseñar y evaluar, d) permite definir la responsabilidad del alumno, del profesor y de la institución con respecto a la sociedad y e) permite establecer un diseño curricular con sentido común. ${ }^{3}$

Por el contrario no indica: a) cómo han de enseñar los profesores, b) como han de aprender los alumnos y c) no presupone ser restrictivo con la metodología.

La definición clara de las competencias permite: a) la organización del currículum, b) el contenido del currículum, c) las asignaturas, d) las estrategias y metodologías docentes, e) los recursos, y f) la evalua- ción y es un buen instrumento para la planificación y el desarrollo de cualquier programa formativo.

\section{ESTRATEGIAS Y METODOLOGÍAS DOCENTES}

Frente a las ideas más tradicionales de que el profesor es el centro del aprendizaje, en la actualidad, se va imponiendo cada vez más la idea de que el centro del aprendizaje ha de ser el alumno. No se trata de enseñar, sino que se trata de aprender y en este sentido aprender solo puede hacerlo quien aprende. Hay que desechar la idea anticuada de que enseñar es sinónimo de aprender. Quien aprende se sitúa como centro del proceso de aprendizaje y el papel del docente es más de facilitador del aprendizaje que de transmisor de conocimientos.

Por ello, a la hora de escoger una determinada estrategia o metodología docente hay que potenciar aquella que permita esta tendencia. Se trata de utilizar aquellas metodologías que aseguren una participación lo más activa posible por parte de los alumnos. En este sentido una de las más útiles es la del aprendizaje basado en la solución de problemas donde el alumno aprende en el contexto de un problema concreto, ${ }^{6,7}$. El aprendizaje basado en la solución de problemas permite un mayor aprendizaje autónomo por parte del estudiante, un aprendizaje más participativo y responsable del alumno, el aprendizaje de aspectos relevantes y la eliminación de aspectos irrelevantes. En este modelo, el profesor asume lo que ha de ser su verdadero rol, es decir el de facilitador del aprendizaje.

En cualquier caso, cuando decidimos utilizar una determinada estrategia y metodología docente hemos de tener evidencias de que es la mejor opción para los fines que perseguimos. Y aquí entramos en un tema que es también objeto de atención por parte de los educadores en Ciencias de la Salud.

Cees Van de Vleuten (Maastricht) escribió la siguiente frase: "A pesar de que los profesores de medicina están formados para tomar decisiones basadas en la evidencia disponible, cuando se ponen el sombrero de profesor, parecen abandonar todo su pensamiento crítico sobre qué funciona y qué no funciona, y se fían de la tradición y la intuición".

Nada más cercano a la realidad. Mayoritariamente la educación ha estado y sigue estando gobernada por la tradición y la intuición. Existe una creencia general entre los investigadores que la evidencia para aceptar o rechazar diferentes enfoques educativos no existe y que existen problemas en la investigación en educación, de medida y de causalidad, que no 
se presentan en las Ciencias de la Salud. Sin embargo se ha demostrado claramente que comparando con las Ciencias de la Salud, la educación se enfrenta a problemas, sino de idéntica, si de similar complejidad.

A la vista de estas consideraciones, y ante el modelo totalmente aceptado de la Medicina basada en la evidencia, los educadores médicos se plantearon por que no aplicar estos mismos principios a la enseñanza. Esta idea hizo surgir un proyecto conocido como BEME (Best evidence based medical education o Educación médica basada en la mejor evidencia) ${ }^{8}$. Este proyecto propugna la utilización por parte de los docentes en su práctica, de métodos y aproximaciones a la educación basados en la mejor evidencia disponible, debiéndose escoger la metodología más adecuada de acuerdo con las mejores evidencias contrastadas que existan sobre su utilidad. Las evidencias pueden encontrarse en la literatura publicada en el campo de la educación y pueden ser de diferentes tipos:

a) Evidencia basada en el juicio profesional

b) Evidencia basada en los principios educativos

c) Evidencia basada en el estudio de casos

d) Evidencia basada en el consenso alcanzado sobre la base de una experiencia

e) Evidencia basada en estudios comparables en otras áreas

f) Evidencia en estudios no experimentales, bien diseñados

g) Evidencia basada en estudios quasi-experimentales bien diseñados

h) Evidencia basada en estudios bien diseñados y controlados

La adopción del concepto de la educación basada en la mejor evidencia no requiere que todo profesor sea un investigador en educación. Se requiere que el profesor sea capaz de apreciar la evidencia disponible y tomar decisiones en base a ella. Es sobre todo una actitud mental. En la medicina y otras áreas académicas, las actividades docentes son menos consideradas que otras actividades como las de investigación. La implicación de los profesores en la investigación para la búsqueda de la mejor evidencia en la educación podría ayudar a resolver este problema.

\section{EL ROL DEL PROFESOR}

Las nuevas estrategias docentes utilizadas hoy día que priman el concepto de la enseñanza centrada en el estudiante, basada en el concepto del Aprender versus el Enseñar y la aplicación de las nuevas tecnologías y de la nuevas técnicas de eva- luación hacen que el docente sienta una cierta sensación de ansiedad, de incerteza, de pérdida de poder e incluso de devaluación de su papel. Ello obliga sin duda a replantearnos el papel actual del docente. El docente, hoy en día no debiera ser un mero transmisor de conocimientos, sino que debe desarrollar nuevos roles o funciones.

Analicemos someramente estas funciones o roles. ${ }^{9}$

\section{El profesor como proveedor de información}

a) El profesor como impartidor de clases magistrales: Tradicionalmente el alumno espera que se le enseñe y cree que la responsabilidad del profesor es transmitirle información, conocimientos y comprensión de un tema determinado. Este nos lleva al tradicional papel del profesor como proveedor de información en el contexto de una clase magistral. El profesor es visto como un experto en conocimientos en su campo y que transmite este conocimiento a través de la palabra. Por otra parte la importancia al papel del profesor como proveedor de información tiene connotaciones culturales.

b) El profesor de clases prácticas: Las clases prácticas son un excelente contexto para la transmisión de información relevante o la adquisición de determinadas habilidades. El profesor en este contexto selecciona, organiza y facilita la información.

\section{El profesor como modelo para el estudiante}

a) El profesor como modelo de profesional: La importancia del profesor como modelo está bien documentada. Los estudiantes en muchos casos aprenden por observación e imitación del profesor y lo hacen sobre todo no de lo que el profesor dice sino de lo que hace y como lo hace. El modelo que da el profesor es el mejor sistema de transmitir valores, actitudes y patrones de razonamiento y de conducta de los estudiantes.

b) El papel de modelo como profesor: Los profesores sirven de modelo a los estudiantes no solo cuando ejercen sus obligaciones profesionales, sino también cuando desarrollan su actividad en la clase, en la tutoría o en el seminario. El buen profesor es aquel que transmite su propio enfoque sobre un determinado problema, discutiéndolo de tal manera que les hace comprender la importancia del mismo.

\section{El profesor como facilitador}

a) Facilitador del aprendizaje: El desplazamiento del centro de gravedad del aprendizaje hacia el estudiante ha provocado o debería provocar también un cambio fundamental en las funciones del profesor, en 
el sentido de verle sobre todo como un facilitador del aprendizaje, aunque hay que reconocer que no todos los profesores se saben adaptar a este papel. La introducción de la enseñanza basada en la resolución de problemas con el consiguiente cambio de la relación profesor-estudiante ha incidido sobre el paso del modelo de profesor como transmisor de información al de facilitador del aprendizaje. En este contexto el papel del profesor no es informar a los estudiantes sino a estimularlos y facilitarles su propio aprendizaje, usando el problema como núcleo del aprendizaje. La creciente disponibilidad de materiales docentes también ha incrementado la necesidad de ver al profesor como un facilitador del aprendizaje. No todos los materiales en cualquiera de sus formatos son adecuados para todos los estudiantes. Es responsabilidad del profesor el facilitar el uso de los recursos más adecuados, resolviendo las deficiencias que puedan presentar y adecuarlos al currículum.

b) El profesor como tutor-mentor: Este papel es otra función del profesor que está de moda. La "mentorización" o tutorización ha de ser vista como una relación especial que se desarrolla entre dos personas en las que el mentor-tutor se convierte en un soporte aunque evitando la dependencia. Es un proceso en la que una persona actúa hacia otra como consejero o guía y que ayuda al aprendizaje mediante una relación de soporte, relación por otra parte que suele ser de larga duración.

\section{El profesor como evaluador}

a) Evaluador de los estudiantes: La evaluación del estudiante es sin duda una las funciones más importantes de las funciones docentes del profesorado. Dice Ian Lang: "I believe that teaching without testing is like cooking without tasting". Evaluar representa una función distinta y potencialmente separada del papel de profesor. De hecho es posible que alguien que sea un profesor experto no sea una examinador experto. Todas las instituciones necesitarían en su "staff" algunos profesores con un especial conocimiento y comprensión de los diferentes métodos de evaluación. Sin embargo, el evaluar debería ser contemplada como una parte integral de las funciones del profesor y parte importante de su dedicación en la educación superior. El papel del profesor como evaluador a menudo es percibido de forma diferente a las otras funciones. Mientras que en los papeles anteriormente descritos, el objetivo del profesor es ayudar al estudiante por diversos mecanismos a alcanzar los objetivos del programa educativo, el papel de evaluador se contempla como una función juzgadora. b) Evaluador del currículum: El profesor ha de ser responsable no solo de planificar o implementar un programa educativo y evaluar al alumno, sino también evaluar el programa y el currículum seguido. Hacer un seguimiento y una evaluación de la eficacia de los programas y de los curricula es reconocido hoy día como una parte integral del proceso educativo. La calidad de la enseñanza y del aprendizaje debe ser evaluada a través del feed-back facilitado por los estudiantes, por los pares y por evaluación del producto final del programa. La evaluación de la enseñanza y del currículum ha de ser realizada a nivel institucional siendo el profesor una de las piezas clave del proceso. El feed-back procedente de diferentes vías debe ser utilizado para identificar aquellas áreas en las cuales se requiere un mejoría y un mayor desarrollo del profesorado.

La autoevaluación del profesor debe ser considerada también como una parte integral de las obligaciones profesionales de los profesores, reconociendo así mismo su responsabilidad para evaluar su propio rendimiento. Forma parte de las funciones del profesorado ser evaluador de su propia competencia. La evaluación del profesor y del currículum es una forma de rendir cuentas al sistema educativo, a la sociedad, a la profesión y a las instancias que financian dicho proceso educativo.

\section{El profesor como planificador}

a) Planificador del currículum: Los profesores deben implicarse en los procesos de planificación curricular La planificación del currículum es una importante función del profesor.

b) Planificador de asignaturas, cursos, etc: El mejor currículum del mundo puede convertirse en ineficaz si las asignaturas que lo integran tienen poca o ninguna relación con el mismo. Tradicionalmente muchos de los currícula actuales están basados en disciplinas, aunque recientemente se están introduciendo currículo integrados o interdisciplinarios. Estos abordajes se deben reflejar en el diseño de las asignaturas. Esta planificación como la del currículum requiere dedicación por parte del profesorado. La participación en la planificación de los cursos y asignaturas da al profesor una oportunidad de ejercer una influencia significativa en el proceso educativo y en el diseño de las actividades docentes que se ajusten a los objetivos de la institución.

\section{El profesor como generador de recursos docentes}

a) Creador de material docente: Hoy día es acep- 


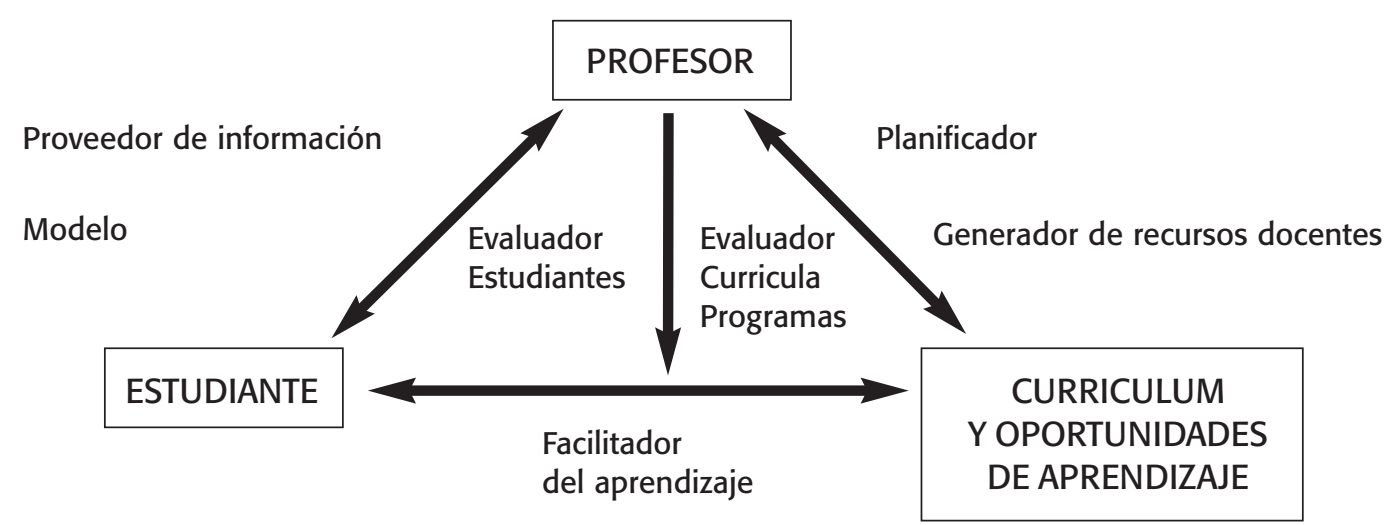

tada de forma general la necesidad de generar recursos docentes. Con la aparición de la estrategias educativas centrada en el estudiante, estos dependen de la disponibilidad de dichos recursos en sus diferentes formatos. Aún en los curricula tradicionales, los estudiantes dedican más tiempo a la consulta de estos recursos que los profesores. El papel del profesor como generador de recursos docentes y de nuevos ambientes de aprendizaje ofrece grandes posibilidades. Por otra parte, la expansión de las nuevas tecnologías han incrementado los formatos de los materiales docentes a los cuales el estudiante puede tener acceso y facilitan que este adquiera más responsabilidad en su propia educación. Como generadores de materiales docentes, los profesores deben estar al día de los cambios en la tecnología implicada. Es necesario que los profesores sean capaces no solo de generar nuevos recursos, sino también de adaptar los existentes a sus necesidades

b) El profesor como productor de guías docentes: Si bien el aprendizaje se ve facilitado por el contacto cara a cara del estudiante y el profesor, también es cierto que el tiempo disponible para ello es limitado. Por ello la confección de guías de estudio preparadas en formato electrónico o en soporte papel se convierten en tutores disponibles para el estudiante a lo largo de las 24 horas del día y están diseñadas para facilitar el aprendizaje. Las guías de estudio especifican al estudiante lo que debe aprender, lo que se espera que sepa hacer al final del curso y las competencias necesarias que debe adquirir. Sin embargo debe tenerse en cuenta que no es útil que el profesor pierda tiempo en producir material docente sobre aspectos que ya están cubiertos por libros u otro material docente.

En resumen todas estas funciones docentes del profesor se esquematizan en la figura 2 .

\section{CONCLUSIONES}

Hemos analizado someramente algunos de los aspectos a tener en cuenta cuando se nos plantea desarrollar un programa educativo. Confiamos que lo expuesto, juntamente con la información que se puede obtener en la bibliografía citada, sea útil a todos los interesados a la hora de elaborar este curso de postgrado sobre Metodología de la Investigación.

\section{BIBLIOGRAFIA}

1. Postgraduate Medical Education. WFME Global Standars for Quality Improvement. World Federation for Medical Education. WFME Office. University of Copenhagen. Denmark, 2003. (www.wfme.org)

2. Harden, R.M., Crosby, J.R.; Davis, M.H. and Friedman M. (1999). From competency to meta-competency: a model for the specification of learning outcomes. Medical Teacher, 21, 6, pp. 546-552

3. The Scottish Dean's Medical Curriculum Group. The Scottish Doctor-learning outcomes for the medical undergraduated in Scotland: a foundation for competent and reflective practitioners. Medical Teacher, 24,2: pp. 126-143.

4. AMEE (1999). Outcome-based medical education. AMEE Medical Education Guide $\mathrm{n}^{\circ} 14$ AMEE Centre for Medical Education, University of Dundee, UK.

5. Harden, R.M., Crosby, J.R., and Davis, M.H. An introduction to outcome-based education. Medical Teacher, 21, 1: pp 7-14.

6. Barrows, HS. y Tamblyn, E.M. (1980). Problem based-learning. An approach to Medical education. New York Springer Publications.

7. Schmidt, HG . (1983). Problem-based learning: rationale and description. J Med Educ 17, 11.

8. Hart, IR. (1999) Best Evidence Medical Education (BEME). Editorial, Medical Teacher, 21, $\mathrm{n}^{\circ} 5$ 453-454.

9. Harden, R. M. and Crosby, J.R. (2000). The Good Teacher is more than a Lecturer: The Twelve Roles of the Teacher. AMEE Medical Education Guide n ${ }^{\circ} 20$. Medical Teacher, 22(4): 334-347. 